\title{
The Synthesis of 7-Substituted-2,3-dihydropyrido [4,3-d]pyridazine-1,4-diones and 1,4-Dioxo-7-substituted- 1,2,3,4-tetrahydropyrido $[4,3-d]$ pyridazine 6-Oxides from Methyl Ketones
}

\author{
Benjamin Prek and Branko Stanovnik \\ Faculty of Chemistry and Chemical Technology, University of Ljubljana, \\ Večna pot 113, P. O. Box 537, 1000 Ljubljana, Slovenia \\ *Corresponding author: E-mail: branko.stanovnik@fkkt.uni-lj.si
}

Received: $12-07-2017$

\begin{abstract}
Dedicated to Professor Emeritus Miha Tišler, University of Ljubljana, on the occasion of his $90^{\text {th }}$ birthday.
\end{abstract}

\begin{abstract}
A general four-step transformation of alkyl, cycloalkyl, aryl, and heteroaryl methyl ketones via 3-(dimethylamino)-1-substituted-prop-2-en-1-ones, followed by microwave [2+2] cycloaddition of dimethyl acetylenedicarboxylate, cyclization of $(2 E, 3 E)-2$-[(dimethylamino)methylene]-3-(2-substituted)succinates with ammonia or hydroxylamine hydrochloride into 2-substituted-pyridine-4,5-dicarboxylates and their $\mathrm{N}$-oxides and final cyclization with hydrazine hydrate into of 7-substituted-2,3-dihydropyrido[3,4-d]pyridazine-1,4-diones and 1,4-dioxo-7-substituted-1,2,3,4-tetrahydropyrido[4,3-d]pyridazine 6-oxides is shown.
\end{abstract}

Keywords: methyl ketones, 7-substituted-2,3-dihydropyrido[4,3-d]pyridazine-1,4-diones, 1,4-dioxo-7-substituted1,2,3,4-tetrahydropyrido[4,3- $d]$ pyridazine 6-oxides

\section{Introduction}

There are several methods for the preparation of 2,3-dihydro[4,3-d]pyridazine-1,4- dione derivatives. They have been prepared by treatment of diethyl or dimethyl pyridine-3.4-dicarboxylate with hydrate of hydrazine in refluxing ethanol, and 6-aryl- and 6-aryl-2-methyl derivatives with hydrate of hydrazine in refluxing ethanol, which allow the formation of the corresponding 7-substituted and 5,7-disubstituted-2,3-dihydropyridazine[3,4-d]pyridazine-1,4-diones. ${ }^{1-4}$ Cyclization of ethyl 3-cyanoisonicotinate with hydrazine proceeds at room temperature to give 4-aminopyrido[3,4-d]pyridazine- $1(2 H)$-one, ${ }^{5,6}$ while pyridine-3,4-dicarbonitriles give the corresponding pyrido[3,4- $d]$ pyridazine-1,4-diones. ${ }^{7}$ Other methods include cycloamination of 4-carbofunctional-5-vinylpyridazines, ${ }^{8,9}$ condensation of 4,5-dicarbofunctional pyridazines with amines, ${ }^{9,10}$ condensation of 4-(iminomethyl)pyridazines with enolates, ${ }^{10}$ intramolecular cyclization of pyridinecarbohydrazides, ${ }^{1,11}$ intramolecular cyclization of 4-vinylpyridazine-5-carbonitriles, ${ }^{12,13}$ by ring enlargement of furo[3,4-c]pyridine-1,3-diones, ${ }^{2,3,5,14,15} 1 \mathrm{H}$-pyrrolo[3,4-c] pyridine-1,3(2H) -diones with hydrazine, ${ }^{1,4,16,17}$ by reaction of $5 \mathrm{H}$-pyrano[ $3,4-d]$ pyridazines with amines, ${ }^{8}$ intramolecular [4+2]cycloaddition of $1,2,4,5$-tetrazines, ${ }^{18}$ ring contraction of $2 H-1,2,4$-triazepines..$^{14}$ For a review see..$^{19}$

Enaminones are well known starting compounds in the synthesis of heterocyclic systems. Their reactivity enables various transformations and functionalizations. Their synthetic value and broad applicability has also been demonstrated in the preparation of natural products and their analogues, such as aplysinopsins, ${ }^{20}$ meridianines, ${ }^{21}$ and dipodazines. ${ }^{22}$ Besides the evident reactions with nucleophiles, they also exhibit reactivity with electrophiles as well, which only adds to their importance as building blocks in organic synthesis. ${ }^{23}$ Reactions with electrophiles have been demonstrated in the synthesis of polysubstituted butadienes by microwave-assisted formal $[2+2]$ cycloadditions of enaminones to electron-poor acetylenes. ${ }^{24}$ 
The functionalized buta-1,3-dienes as the basis of the synthetic route presented in this paper are prepared from simple and commercially available compounds such as alkyl, aryl, and heteroaryl methyl ketones. These are transformed by treatment with $\mathrm{N}, \mathrm{N}$-dimethylformamide dimethyl acetal (DMFDMA) or tert-butoxybis(dimethylamino)methane (Bredereck's reagent) into the corre- sponding 3-(dimethylamino)-1-substituted-prop-2-enones, which are further transformed in a regiospecific microwave assisted $[2+2]$ cycloaddition with dimethyl acetylenedicarboxylate (DMAD) $)^{25}$ to the before mentioned 1,3-butadienes. These highly functionalized buta-1,3dienes proved to be useful and versatile reagents in the formation of highly substituted pyridine, pyridine $\mathrm{N}$-oxides,

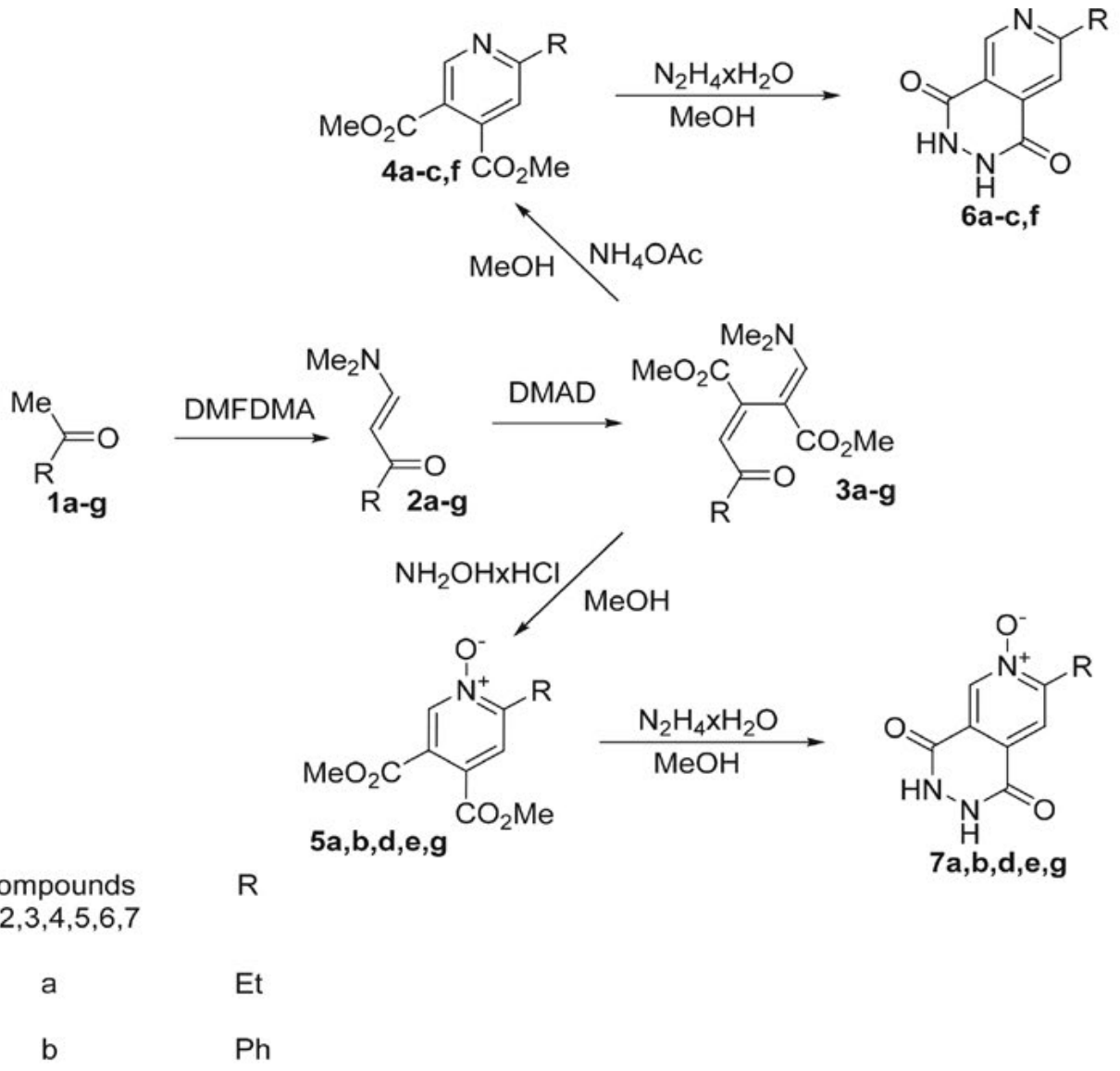

Table 1.

Compound $\quad$ Reaction time [h] Yield [\%]

$6 a \quad 857$

$\begin{array}{lll}6 b & 24 & 20\end{array}$

$\begin{array}{lll}6 c & 12 & 74\end{array}$

6f $\quad 15 \quad 44$

$\begin{array}{lll}7 a & 7 & 23\end{array}$

$7 \mathrm{~b}-12 \quad 90$

$\begin{array}{lll}7 d & 4 & 75\end{array}$

$\begin{array}{lll}7 \mathrm{e} & 4 & 89\end{array}$

Scheme 1. Preparation of 7-substituted-2,3-dihydropyrido[3,4- $d$ ]pyridazine-1,4-diones 6a-c,f and 1,4-dioxo-7-substituted-1,2,3,4-tetrahydropyrido[4,3-d]pyridazine 6-oxides $7 \mathbf{a}, \mathbf{b}, \mathbf{d}, \mathbf{e}, \mathbf{g}$ from methyl ketones $\mathbf{1 a - g}$. 
pyrrole, pyrido[3,4-c]pyridazine derivatives, ${ }^{25 b} 2$-substituted pyridine-3,4-dicarboxylates and their $\mathrm{N}$-oxides, ${ }^{10 \mathrm{~g}}$ and triazafulvalene derivatives. ${ }^{25 d}$

Polysubstituted aminobutadienes, prepared by this procedure, are suitable for the preparation of polysubstituted pyridine derivatives. They also represent a group of isomeric intermediates in regard to the aminobutadienes prepared via the Michael addition in the Bohlmann-Rahtz synthesis of pyridine derivatives. ${ }^{26}$ On this basis, a simple metal-free synthesis of 2-alkyl-, 2-cycloalkyl-, 2-aryl-, and 2-heteroaryl-substituted pyridine-3,4-dicarboxylates and their $\mathrm{N}$-oxides has also been reported. ${ }^{25 g}$ Recently, we reported on a simple one-pot metal-free synthesis of 2,4,5-trisubstituted pyridine derivatives and their $\mathrm{N}$-oxides by $[2+2]$ cycloaddition of propyne iminium salts as electron-poor acetylenes to enaminones as well. ${ }^{27} \mathrm{We}$ also reported a simple, metal-free synthesis of electron rich 2,4,6-trisubstituted pyridine derivatives ${ }^{28}$ and the synthesis of polysubstituted benzene derivatives, where $\mathrm{N}, \mathrm{N}$-dimethylacetamide dimethyl acetal (DMADMA) served as the reagent and building block for generating aromatic final products. ${ }^{29,30}$ Our existing knowledge of the enaminones and 1,3-butadienes has been expanded to the synthesis of pyridines starting from Boc-protected amino acids, and the results of our research are presented in this paper.

\section{Results and Discussion}

In this communication we report a general and simple synthesis of 7-substituted-2,3-dihydropyrido[3,4-d] pyridazine-1,4-diones $\mathbf{6 a - c , f}$ and 1,4-dioxo-7-substituted-1,2,3,4-tetrahydropyrido[4,3-d]pyridazine 6-oxides 7a, b,d,e,g from methyl ketones 1a-g. We have reported earlier a simple metal-free synthesis of dimethyl 2-substituted-pyridine-4,5-dicarboxylates $\mathbf{4 a - c , f}$ and their $\mathrm{N}$-oxides $\mathbf{5 a}, \mathbf{b}, \mathbf{d}, \mathbf{e}, \mathbf{g}$ in the following manner: alkyl, aryl, and heteroaryl ketones 1a-g have been converted by treatment with $\mathrm{N}, \mathrm{N}$-dimethylformamide dimethylacetal /DMFDMA) or $t$-butoxybis(dimethylamino)methane (Bredereck's reagent) into the corresponding 3-(dimethylamino)-1-substituted-prop-2-en-ones $\mathbf{2 a - g}$, followed by microwave assisted $[2+2]$ cycloaddition to dimethyl acetylenedicarboxylate to give dimethyl $(2 E, 3 E)-2-\{$ (dimethylamino)methylene\}-3-(2-substituted)succinates 3a-g. Compounds 3 gave by treatment with ammonium acetate or hydroxylamine hydrochloride the corresponding dimethyl 2-substituted-pyridine-4,5-dicarboxylates $\mathbf{4 a - c , f}$ and dimethyl 2-substituted-4,5-bis(methoxycarbonyl)pyridine $N$-oxides $\mathbf{5 a}, \mathbf{b}, \mathbf{d}, \mathbf{e}, \mathbf{g} .{ }^{25, f, g}$ Treatment of compounds $\mathbf{4}$ and 5 with hydrazine hydrate afforded 7-substituted-2,3-dihydropyrido[3,4-d]pyridazine-1,4-diones 6a-c,f and 1,4-dioxo-7-substituted-1,2,3,4-tetrahydropyrido [4,3-d]pyridazine 6-oxides 7a,b,d,e,g, respectively. (Scheme 1, Table 1).

\section{Experimental}

\section{1. General}

Melting points were determined on a Stanford Research Systems MPA100 OptiMelt automated melting point system. The NMR spectra were obtained on a Bruker Avance DPX 300 at $300 \mathrm{MHz}$ for ${ }^{1} \mathrm{H}$ and $75.5 \mathrm{MHz}$ for ${ }^{13} \mathrm{C}$ and on a Bruker Avance III UltraShield 500 plus at 500 $\mathrm{MHz}$ for ${ }^{1} \mathrm{H}$ and $126 \mathrm{MHz}$ for ${ }^{13} \mathrm{C}$, using acetone- $d_{6}$, acetonitrile- $d_{3}, \mathrm{CDCl}_{3}$, and DMSO- $d_{6}$ with $\mathrm{Me}_{4} \mathrm{Si}$ as the internal standard, as solvents. Mass spectra were recorded on a Agilent 6224 Accurate Mass TOF LC/MS spectrometer, IR spectra on a Perkin Elmer Spectrum BX FTIR spectrophotometer. Column chromatography (CC) was performed on silica gel (Fluka, Silica gel 60, particle size 35-70 $\mu \mathrm{m}$ ).

The preparation of 2-substituted-pyridine-4,5-dicarboxylates $4 \mathbf{a}-\mathbf{c}, \mathbf{f}$ and dimethyl 2-substituted-4,5-bis(methoxycarbonyl)pyridine $\mathrm{N}$-oxides $\mathbf{5 a}, \mathbf{b}, \mathbf{d}, \mathbf{e}, \mathbf{g}$ from alkyl, cycloalkyl, aryl and heteroaryl methyl ketones has been previosly reported in our laboratory. ${ }^{25 e, f, g}$

\section{2. General procedure for the preparation of 7-substituted-2,3-dihydropyrido [3,4-d]pyridazine-1,4-diones and 1,4-dioxo-7-substituted-1,2,3,4- tetrahydropyrido $[4,3-d]$ pyridazine 6-oxides}

To a solution of $0.5 \mathrm{mmol}$ of the starting compound (dimethyl 6-substituted pyridine-3,4-dicarboxylate or 2 -substituted-4,5-bis(methoxycarbonyl)pyridine- $\mathrm{N}$-oxide) in 2-3 mL of methanol, $1 \mathrm{mmol}$ (2 equivalents) of hydrazine monohydrate was added, followed by the addition of 2-3 drops of concentrated hydrochloric acid. The reaction mixture was stirred vigorously and heated to reflux temperature for $4-24 \mathrm{~h}$.

\section{2. 1. 7-Ethyl-2,3-dihydropyrido[4,3-d] pyridazine-1,4-dione $(6 a)$}<smiles>CCc1cc2c(=O)[nH][nH]c(=O)c2cn1</smiles>

The product was preparea Irom dimethyl 6-ethylpyridine-3,4-dicarboxylate (4a,112 $\mathrm{mg}, 0.5 \mathrm{mmol}$ ), 90 ${ }^{\circ} \mathrm{C}, 8 \mathrm{~h}$. The yellow product was collected by vacuum filtration and washed with $\mathrm{Et}_{2} \mathrm{O}$. Yield: $57 \%$ (55 mg), yellow solid; $\mathrm{mp}=$ higher than $350{ }^{\circ} \mathrm{C} .{ }^{1} \mathrm{H}$ NMR $(500 \mathrm{MHz}$, DMSO-d $\left.\mathrm{d}_{6}\right): \delta 1.30\left(3 \mathrm{H}, \mathrm{t}, J=7.5 \mathrm{~Hz}, \mathrm{CH}_{3}\right) ; 2.97(2 \mathrm{H}, \mathrm{q}, J=$ $\left.7.6 \mathrm{~Hz}, \mathrm{CH}_{2}\right) ; 7.74(1 \mathrm{H}, \mathrm{s}, 8-\mathrm{CH}) ; 9.25(1 \mathrm{H}, \mathrm{s}, 5-\mathrm{CH}) .{ }^{13} \mathrm{C}$ NMR $\left(125 \mathrm{MHz}\right.$, DMSO- $\left.d_{6}\right): \delta 13.54,30.77,115.03$, 148.30, 148.47, 164.77, 166.61, 166.74, 168.39. EI-HRMS: $m / z=192.0765\left(\mathrm{MH}^{+}\right)$found; $\mathrm{C}_{9} \mathrm{H}_{10} \mathrm{~N}_{3} \mathrm{O}_{2}$ calculated: $\mathrm{m} / z$ 
$=192.0768\left(\mathrm{MH}^{+}\right)$; IR (ATR): $v$ 3426, 3302, 3250, 2960, $1664,1612,1575,1476,1364,1207,1077,899 \mathrm{~cm}^{-1}$.

\section{2. 2. 7-Phenyl-2,3-dihydropyrido[4,3-d] pyridazine-1,4-dione $(6 \mathrm{~b})$}<smiles>O=c1[nH][nH]c(=O)c2cc(-c3ccccc3)ncc12</smiles>

The product was prepared from dimethyl 6-phenylpyridine-3,4-dicarboxylate (4b, $261 \mathrm{mg}, 096 \mathrm{mmol}$ ), 90 ${ }^{\circ} \mathrm{C}, 12 \mathrm{~h}$. The yellow product was collected by vacuum filtration and washed by Et $\mathrm{O}_{2}$. Yield: $74 \%$ (170 mg), yellow solid; $\mathrm{mp}=335-339{ }^{\circ} \mathrm{C} .{ }^{1} \mathrm{H}$ NMR $\left(500 \mathrm{MHz}, \mathrm{DMSO}-d_{6}\right): \delta$ 7.48-7.57 (3H, m, Ph); 8.19-8.23 (2H, m, Ph); 8.35 (1H, s, $8-\mathrm{CH}) ; 9.36\left(1 \mathrm{H}, \quad\right.$ s, 5-CH). ${ }^{13} \mathrm{C}$ NMR $(125 \mathrm{MHz}$, DMSO-d $)_{6}: \delta 113.8,121.9,126.9,128.9,129.6,135.4,137.8$, 149.1, 155.7, 156.2, 157.5. EI-HRMS: $m / z=240.0767$ $\left(\mathrm{MH}^{+}\right)$found; $\mathrm{C}_{13} \mathrm{H}_{10} \mathrm{~N}_{3} \mathrm{O}_{2}$ calculated: $\mathrm{m} / z=240.0768$ $\left(\mathrm{MH}^{+}\right)$; IR (ATR): $v$ 3289, 3178, 3034, 2978, 2920, 2798, 1898, 1648, 1559, 1454, 1112, $852 \mathrm{~cm}^{-1}$. LC-MS; $9.1 \mathrm{~min}$; $m / z: 240.1\left(\mathrm{MH}^{+}\right)$.

\section{2. 3. 7-Cyclopentenyl-2,3-dihydropyrido $[4,3-d]$ pyridazine-1,4-dione (6c)}<smiles>O=c1[nH][nH]c(=O)c2cc(C3=CCCC3)ncc12</smiles>

The product was prepared from dimethyl 6-cyclopentenylpyridine-3,4-dicarboxylate $\quad(4 \mathrm{c}, 150 \mathrm{mg}, \quad 0.56$ $\mathrm{mmol}), 90^{\circ} \mathrm{C}, 24 \mathrm{~h}$. The solid product that was formed during the reaction was a mixture of the starting dicarboxylate and the product. The solid was collected by vacuum filtration, suspended in chloroform. The insoluble product was once more collected by vacuum filtration. Yield: $20 \%$ (26 mg), brown solid; $\mathrm{mp}=$ higher than $330{ }^{\circ} \mathrm{C} .{ }^{1} \mathrm{H}$ NMR (500 MHz, DMSO- $\left.d_{6}\right): \delta 2.03\left(2 \mathrm{H}, \mathrm{p}, J=7.5 \mathrm{~Hz}, 4-\mathrm{CH}_{2}\right)$; 2.54-2.60 (2H, m, 3-CH $)$; 2.76-2.82 (2H, m, 5- $\left.\mathrm{CH}_{2}\right) ; 6.87$ $(1 \mathrm{H}, \mathrm{s}, 2-\mathrm{CH})$; $7.81(1 \mathrm{H}, \mathrm{s}, 8-\mathrm{CH}) ; 9.23(1 \mathrm{H}, \mathrm{s}, \mathrm{CH}) .{ }^{13} \mathrm{C}$ NMR (125 MHz, DMSO-d $)$ ) $\delta 22.8,32.0,33.2,113.4$, $121.0,133.5,134.5,142.6,148.6,155.2,155.8,156.0$. EI-HRMS: $m / z=230.0915\left(\mathrm{MH}^{+}\right)$found; $\mathrm{C}_{12} \mathrm{H}_{12} \mathrm{~N}_{3} \mathrm{O}_{2}$ calculated: $m / z=230.0924\left(\mathrm{MH}^{+}\right)$. IR (ATR): $v 3163,3016$, 2947, 2892, 2839, 1649, 1598, 1553, 1434, 1368, 1325, 1291, $1232,1101,1036,823 \mathrm{~cm}^{-1}$.

\section{2. 4. 7-(Thiazol-2-yl)-2,3-dihydropyrido[4,3-d] pyridazine-1,4-dione $(6 \mathrm{f})$}<smiles>O=c1[nH][nH]c(=O)c2cc(-c3nccs3)ncc12</smiles>

The product was prepared from dimethyl 6-(thiazol2-yl)pyridine-3,4-dicarboxylate (4f, $89 \mathrm{mg}, 0.32 \mathrm{mmol}$ ), $90{ }^{\circ} \mathrm{C}, 15 \mathrm{~h}$. The yellow product was collected by vacuum filtration and washed with $\mathrm{Et}_{2} \mathrm{O}$. Yield: $44 \%$ ( $35 \mathrm{mg}$ ), yellow solid; $\mathrm{mp}=$ the product decomposes at $250{ }^{\circ} \mathrm{C} .{ }^{1} \mathrm{H}$ NMR $\left(500 \mathrm{MHz}\right.$, DMSO- $\left.d_{6}\right): \delta 7.97(1 \mathrm{H}, \mathrm{d}, J=3.2 \mathrm{~Hz}$, $\mathrm{CH}) ; 8.09(1 \mathrm{H}, \mathrm{d}, J=3.2 \mathrm{~Hz}, \mathrm{CH}) ; 8.54(1 \mathrm{H}, \mathrm{d}, J=1.0 \mathrm{~Hz}$, 8-CH); $9.29(1 \mathrm{H}, \mathrm{d}, J=1.0 \mathrm{~Hz}, 5-\mathrm{CH}) .{ }^{13} \mathrm{C} \mathrm{NMR}(125 \mathrm{MHz}$, DMSO-d $\left.{ }_{6}\right): \delta 113.3,117.2,123.6,123.8,144.9,149.5,151.8$, 155.9, 165.8, 167.6. EI-HRMS: $m / z=245.015\left(\mathrm{MH}^{-}\right)$ found; $\mathrm{C}_{10} \mathrm{H}_{5} \mathrm{~N}_{4} \mathrm{O}_{2} \mathrm{~S}$ calculated: $m / z=245.0139\left(\mathrm{MH}^{-}\right)$; IR (ATR): $v$ 3420, 3260, 1741,1721, 1661, 1654, 1602, 1569, 1465, 1438, 1298, 1242, 1134, 1085, $958 \mathrm{~cm}^{-1}$. LC-MS: 7.1 $\min ; m / z: 247.3\left(\mathrm{MH}^{+}\right)$.

\section{2. 5. 7-Ethyl-1,4-dioxo-1,2,3,4-tetrahydropyrido $[4,3-d]$ pyridazine 6-oxide (7a)}<smiles>CCc1cc2c(=O)[nH][nH]c(=O)c2c[n+]1[O-]</smiles>

The product was prepared from 2-ethyl-4,5-bis(methoxycarbonyl)pyridine 1 -oxide (5a, $156 \mathrm{mg}, 0.65 \mathrm{mmol}$ ), $90{ }^{\circ} \mathrm{C}, 7 \mathrm{~h}$. The yellow solid was collected by vacuum filtration and washed with $\mathrm{Et}_{2} \mathrm{O}$. Yield: $23 \%(31 \mathrm{mg})$, yellow solid; $\mathrm{mp}=270-276{ }^{\circ} \mathrm{C} .{ }^{2} \mathrm{H}$ NMR $\left(500 \mathrm{MHz}, \mathrm{DMSO}-d_{6}\right): \delta$ $1.26\left(3 \mathrm{H}, \mathrm{t}, J=7.4 \mathrm{~Hz}, \mathrm{CH}_{3}\right) ; 2.89\left(2 \mathrm{H}, \mathrm{q}, J=7.4 \mathrm{~Hz}, \mathrm{CH}_{2}\right)$; $7.89(1 \mathrm{H}, \mathrm{s}, 8-\mathrm{CH}) ; 8.61(1 \mathrm{H}, \mathrm{s}, 5-\mathrm{CH}) .{ }^{13} \mathrm{C}$ NMR $(125$ MHz, DMSO-d $\left.{ }_{6}\right): \delta 10.5,23.3,120.2,123.5,125.0,134.8$, 153.7, 154.3, 156.0. EI-HRMS: $m / z=208.0712\left(\mathrm{MH}^{+}\right)$ found; $\mathrm{C}_{9} \mathrm{H}_{10} \mathrm{~N}_{3} \mathrm{O}_{3}$ calculated: $m / z=208.0717\left(\mathrm{MH}^{+}\right)$. IR (ATR): $v 3203,3047,2974,2810,1626,1549,1454,1432$, $1366,1317,1268,1043,814 \mathrm{~cm}^{-1}$.

\section{2. 6. 1,4-Dioxo-7-phenyl-1,2,3,4-tetrahydropyrido $[4,3-d]$ pyridazine 6-oxide (7b)}<smiles>O=c1[nH][nH]c(=O)c2c[n+]([O-])c(-c3ccccc3)cc12</smiles>

The product was prepared from 4,5-bis(methoxycarbonyl)-2-phenylpyridine 1-oxide (5b, $241 \mathrm{mg}, 0.84 \mathrm{mmol}$ ), $90{ }^{\circ} \mathrm{C}, 12 \mathrm{~h}$. The yellow solid was collected by vacuum filtration and washed with $\mathrm{Et}_{2} \mathrm{O}$. Yield: $90 \%$ (190 mg), yellow 
solid; $\mathrm{mp}=275-285^{\circ} \mathrm{C} .{ }^{1} \mathrm{H}$ NMR $\left(500 \mathrm{MHz}, \mathrm{DMSO}-d_{6}\right): \delta$ 7.49-7.53 (3H, m, Ph); 7.82-7.85 (2H, m, Ph); 7.95 (1H, s, $8-\mathrm{CH}) ; 8.65(1 \mathrm{H}, \quad \mathrm{s}, \quad 5-\mathrm{CH}) .{ }^{13} \mathrm{C} \mathrm{NMR}(125 \mathrm{MHz}$, DMSO-d $)_{6}: \delta 123.1,124.3,126.1,127.9,129.3,129.6,131.9$, 135.9, 150.4, 153.9, 154.7. EI-HRMS: $m / z=256.0716$ $\left(\mathrm{MH}^{+}\right)$found; $\mathrm{C}_{13} \mathrm{H}_{10} \mathrm{~N}_{3} \mathrm{O}_{3}$ calculated: $\mathrm{m} / z=256.0717$ $\left(\mathrm{MH}^{+}\right)$. IR (ATR): $v$ 3338, 3066, 2861, 1807, 1657, 155, 1466, 1448, 1269, 1096, $813 \mathrm{~cm}^{-1}$. LC-MS: $7.4 \mathrm{~min} ; \mathrm{m} / z$ : $256.1\left(\mathrm{MH}^{+}\right)$.

\section{2. 7. 7-(1-Methyl-1H-pyrrol-2-yl)-1,4-dioxo- 1,2,3,4-tetrahydropyrido $[4,3-d]$ pyridazine 6-oxide (7d)}<smiles>Cn1cccc1-c1cc2c(=O)[nH][nH]c(=O)c2c[n+]1[O-]</smiles>

The product was prepared from 4,5-bis(methoxycarbonyl)-2-(1-methyl-1H-pyrrol-2-yl)pyridine 1-oxide (5d, $173 \mathrm{mg}, 0.6 \mathrm{mmol}), 90^{\circ} \mathrm{C}, 4 \mathrm{~h}$. The yellow product was collected by vacuum filtration and washed with $\mathrm{Et}_{2} \mathrm{O}$. Yield: $75 \%(115 \mathrm{mg})$, yellow solid; $\mathrm{mp}=222-238^{\circ} \mathrm{C} .{ }^{1} \mathrm{H}$ NMR $\left(500 \mathrm{MHz}\right.$, DMSO- $\left.d_{6}\right): \delta 3.58\left(3 \mathrm{H}, \mathrm{s}, \mathrm{CH}_{3}\right) ; 6.12(1 \mathrm{H}$, dd, $\left.J_{1}=3.7 \mathrm{~Hz}, J_{2}=2.6 \mathrm{~Hz}, 3-\mathrm{CH}\right) ; 6.40\left(1 \mathrm{H}, \mathrm{dd}, J_{1}=3.7\right.$ $\left.\mathrm{Hz}, J_{2}=1.8 \mathrm{~Hz}, 5-\mathrm{CH}\right) ; 7.00(1 \mathrm{H}$, deg. dd, $J=2.2 \mathrm{~Hz}, 4-$ $\mathrm{CH}) ; 7.85(1 \mathrm{H}, \mathrm{s}, 8-\mathrm{CH}) ; 8.64(1 \mathrm{H}, \mathrm{s}, 5-\mathrm{CH}) .{ }^{13} \mathrm{C}$ NMR $(125$ MHz, DMSO-d $\left.{ }_{6}\right): \delta 40.5,112.9,118.3,129.6,129.8,130.1$, 131.0, 131.7, 141.1, 149.9, 159.9, 160.6. EI-HRMS: $m / z=$ 259.0822 $\left(\mathrm{MH}^{+}\right)$found; $\mathrm{C}_{12} \mathrm{H}_{11} \mathrm{~N}_{4} \mathrm{O}_{3}$ calculated: $\mathrm{m} / z=$ 259.0826 $\left(\mathrm{MH}^{+}\right)$; IR (ATR): v 3424, 3301, 3078, 2953, 2929, $1664,1651,1558,1485,1471,1308,1255,1103,1072,822$ $\mathrm{cm}^{-1}$. LC-MS: $7.3 \mathrm{~min} ; \mathrm{m} / z: 259.2\left(\mathrm{MH}^{+}\right)$.

\section{2. 8. 7-(1-Methyl-1H-pyrrol-3-yl)-1,4-dioxo-1,2,3,4-tet-} rahydropyrido $[4,3-d]$ pyridazine 6-oxide $(7 \mathrm{e})$

The product was<smiles>Cn1ccc(-c2cc3c(=O)[nH][nH]c(=O)c3c[n+]2[O-])c1</smiles>

$\left(\mathrm{MH}^{+}\right)$found; $\mathrm{C}_{12} \mathrm{H}_{11} \mathrm{~N}_{4} \mathrm{O}_{3}$ calculated: $\mathrm{m} / \mathrm{z}=259.0826$ $\left(\mathrm{MH}^{+}\right)$; IR (ATR): $v$ 3416, 3295, 3199, 2992, 1653, 1614, $1571,1535,1487,1442,1366,1250,1171,1091,831 \mathrm{~cm}^{-1}$. LC-MS: $6.6 \mathrm{~min} ; \mathrm{m} / z: 259.2\left(\mathrm{MH}^{+}\right)$.

\section{2. 9. 1,4-Dioxo-7-(pyrazin-2-yl)-1,2,3,4- tetrahydropyrido $[4,3-d]$ pyridazine 6-oxide $(7 \mathrm{~g})$}<smiles>O=c1[nH][nH]c(=O)c2c[n+]([O-])c(-c3cnccn3)cc12</smiles>

The product was prepared from 4,5-bis(methoxycarbonyl)-2-(pyrazin-2-yl)pyridine 1-oxide (5g,144 mg, 0.5 $\mathrm{mmol}), 90^{\circ} \mathrm{C}, 24 \mathrm{~h}$. The yellow product was collected by vacuum filtration and washed with $\mathrm{Et}_{2} \mathrm{O}$. Yield: $95 \%$ (125 $\mathrm{mg})$, yellow solid; $\mathrm{mp}=$ higher than $350{ }^{\circ} \mathrm{C} .{ }^{1} \mathrm{H}$ NMR $(500$ $\left.\mathrm{MHz}, \mathrm{DMSO}-d_{6}\right): \delta 8.56(1 \mathrm{H}, \mathrm{s}, \mathrm{CH}) ; 8.72(\mathrm{~s}, 1 \mathrm{H}, \mathrm{CH})$; $8.78(1 \mathrm{H}, \mathrm{d}, J=2.5 \mathrm{~Hz}, \mathrm{CH}) ; 8.87-8.91(1 \mathrm{H}, \mathrm{m}, \mathrm{CH}) ; 9.87$ $(1 \mathrm{H}, \mathrm{d}, J=1.5 \mathrm{~Hz}, \mathrm{CH}) .{ }^{13} \mathrm{C}$ NMR $\left(125 \mathrm{MHz}, \mathrm{DMSO}-\mathrm{d}_{6}\right): \delta$ $124.1,136.5,144.6,144.9,145.26,145.29,145.6,146.9$, 157.0, 158.1. EI-HRMS: $m / z=258.0624\left(\mathrm{MH}^{+}\right)$found; $\mathrm{C}_{11} \mathrm{H}_{8} \mathrm{~N}_{5} \mathrm{O}_{3}$ calculated: $m / z=258.0622\left(\mathrm{MH}^{+}\right)$; IR (ATR): $v$ 3447, 3033, 2981, 1667, 1635, 1563, 1498, 1404, 1273, 1242, $1119,827 \mathrm{~cm}^{-1}$. LC-MS: $5.9 \mathrm{~min} ; \mathrm{m} / z: 255.7$ [(M-2H $\left.)^{-}\right]$.

\section{Conclusion}

A general four-step metal-free synthesis of a series of 7-substituted-2,3-dihydropyrido[3,4- $d]$ pyridazine-1,4-diones and 1,4-dioxo-7-substituted-1,2,3,4-tetrahydropyrido[4,3-d]pyridazine 6-oxides was designed starting from alkyl, cycloalkyl, aryl or heteroaryl methyl ketones in good to excellent yields.

\section{Acknowledgement}

Financial support from the Slovenian Research Agency through grants P0-0502-0103, P1-0179 and J16689-0103-04 are gratefully acknowledged. We also thank the Krka d.d. (Novo mesto, Slovenia) for financial support.

\section{Reference}

1. Y. Oka, K. Omura, A. Miyake, K. Itoh, M. Tomimoto, N. Tada, S. Yurugi, Chem. Pharm Bull. 1975, 23, 2239-2250.

2. R. G. Jones, J. Am. Chem. Soc. 1956, 78, 159-163.

3. D. B. Paul, Aust. J. Chem. 1984, 37, 87-93.

4. T. J. Van Bergen, R. M. Kellogg, J. Amer. Chem. Soc. 1972, 94, 8451-8471. 
5. I. Matsuura, K. Okui, Chem. Pharm Bull. 1969, 17, 2266-2272.

6. K. Yoshida, H. Otomasu, Yakugaku Zasshi 1976, 96, 33-36; Chem. Abstr. 1976, 84, 121754.

7. F. Ishikawa, S. Miyazaki, K. Ueno, JP 46029876 (1971); Chem. Abst. 1971, 75, 140875.

8. P. Y. Boamah, N. Haider, G. Heinisch, J. Moshuber, J. Heterocycl. Chem. 1988, 25, 879-883.

9. P. Y. Boamah, N. Haider, G. Heinisch, J. Heterocycl. Chem. 1989, 26, 933-939.

10. G. Heinisch, T. Langer, J. Tonnel, J. Heterocycl. Chem. 1996, 33, 1731-1735.

11. J. Barluenga, M. J. Iglesias, V. Gotor, Synthesis 1987, 662-664.

12. H. Al-Awadhi, F. Al-Omran, M. H. Elnagdi, L. Infantes, C. Foces-Foces, N. Jagorevic, J. Elguero, Tetrahedron 1995, 51, 12745-12762.

13. F. M. Manhi, S. E. Zayed, F. A. Ali, M. H. Elnagdi, Collect. Czech. Chem. Commun.1992, 57, 1770-1774.

14. A. Hasnaoui, M. El Messaoudi, J.-P. Lavergne, J. Heterocycl. Chem. 1985, 22, 25-27.

15. K. J. Gould, N. P. Hacker, J. F. W. McOmie, D. H. Perry, J. Chem. Soc., Perkin Trans.1 1980, 1834-1840.

16. J. Z. Brzezinski, H. B. Bzowski, J. Epsztajn, Tetrahedron 1996, 52, 3261-3272.

17. H. Šladowska, J. Potoczek, M. Sokowska, G. Rajtar, M. Sieklucka-Dziuba, T. Kocki, Z. Kleinrok, Farmaco 1998, 53, 468-474; Chem. Abstr. 1999, 130, 110222.

18. N. Heider, K. Mereiter, R. Wanko, Heterocycles 1994, 38, 1845-1858.

19. M. Sako, Product Class18: Product Subclass 5: Pyridopyridazines. In: Houben-Weyl, Science of Synthesis, Georg Thime Verlag, Stuttgart, New York 2006, vol 16, pp. 1137-1153.

20. (a) Selič, L.; Jakše, R.; Lampič, K.; Golič, L.; Golič-Grdadolnik, S.; Stanovnik, B. Helv. Chim. Acta 2000, 83, 2802-2811; (b) Selič, L.; Stanovnik, B. Tetrahedron 2001, 57, 3159-3164.

21. Jakše, R.; Svete, J.; Stanovnik, B.; Golobič, A. Tetrahedron 2004, 60, 4601-4608; (b) Časar, Z.; Bevk, D.; Svete, J.; Stanovnik, B. Tetrahedron 2005, 61, 7508-7519.

22. (a) Wagger, J.; Bevk, D.; Meden, A.; Svete, J.; Stanovnik, B. Helv. Chim. Acta 2006, 89, 240-248; (b) Wagger, J.; Golič Grdadolnik, S.; Grošelj, U.; Meden, A.; Svete, J.; Stanovnik, B.
Tetrahedron: Asymmetry 2007, 18, 464-475; (c) Wagger, J.; Grošelj, U.; Meden, A.; Svete, J.; Stanovnik, B. Tetrahedron 2008, 64, 2801-2815.

23. (a) Stanovnik, B. J. Heterocycl. Chem. 1999, 36, 1581-1593; (b) Stanovnik, B.; Svete, J. Synlett 2000 1077-1091; (c) Stanovnik, B; Svete, J. Targets in Heterocyclic Systems, Synthesis, Reactions and Properties, (Eds. O. A. Attanasi, D. Spinelli), Italian Society of Chemistry, Rome 2000, Vol. 4, p. 105-137; (d) Stanovnik, B.; Svete, J. Chem. Rev. 2004, 104, 2433-2480.

24. (a) Bezenšek, J.; Koleša, T.; Grošelj, U.; Meden, A.; Stare, K.; Svete, J.; Stanovnik, B. Curr. Org. Chem. 2011, 15, 2530-2539; (b) Bezenšek, J.; Koleša, T.; Grošelj, U.; Wagger, J.; Stare, K.; Meden, A.; Svete, J.; Stanovnik, B. Tetrahedron Lett. 2010, 51, 3392-3397.

25. (a) Uršič, U.; Grošelj, U.; Meden, A.; Svete, J.; Stanovnik, B. Tetrahedron Lett. 2008, 49, 3775-3778; (b) Uršič, U., Svete, J.; Stanovnik, B. Tetrahedron 2008, 64, 9937-9946; (c) Uršič, U.; Grošelj, U.; Meden, A.; Svete, J., Stanovnik, B. Helv. Chim. Acta 2009, 92, 481-490; (d) Uršič, U.; Svete, J.; Stanovnik, B. Tetrahedron 2010, 66, 4346-4356; (e) Bezenšek, J.; Koleša, T.; Grošelj, U.; Meden, A.; Stare, K.; Svete, J.; Stanovnik, B. Curr. Org. Chem. 2011, 15, 2530-2539; (f) Bezenšek, J.; Koleša, T.; Grošelj, U.; Wagger, J.; Stare, K.; Meden, A.; Svete, J.; Stanovnik, B.;Tetrahedron Lett.. 2010, 51, 3392-3397; (g) Bezenšek, J.; Prek, B.; Grošelj, U.; Kasunič, M.; Svete, J.; Stanovnik, B. Tetrahedron 2012, 68, 4719-4731.

26. (a) Bohlmann, F.; Rahtz, D. Chem. Ber. 1957, 90, 2265-2272; (b) Bagley, M. C.; Dale, J. W.; Bower, J. Synlett 2001, 11491151.

27. Bezenšek, J.; Prek, B.; Grošelj, U.; Golobič A.; Stare, K.; Svete, J.; Kantlehner, W.; Maas, G.; Stanovnik, B. Z. Naturforsch. 2014, 69b, 554-566.

28. Prek, B.; Grošelj, U.; Kasunič, M.; Zupančič, S.; Svete, J.; Stanovnik, B. Aust. J. Chem. 2015, 68, 184-195.

29. Prek, B.; Bezenšek, J.; Kasunič, M.; Grošelj, U.; Svete, J.; Stanovnik, B. Tetrahedron 2014, 70, 2359-2369.

DOI:10.1016/j.tet.2014.02.039

30. For a review see: Stanovnik, B. Org. Prep. Proc. Int. 2014, 46, 24-65.

\section{Povzetek}

V tem članku je opisana štiristopenjska pretvorba alkil, cikloalkil, aril in heteroaril metil ketonov, ki jih preko 3-(dimetilamino)-1-substituiranih-prop-2-en-1-onov $\mathrm{z}[2+2]$ cickloadicijo na dimetil acetilendikarboksilat pretvorimo $\mathrm{v}$ (2E,3E)-2-[(dimetilamino)metilen]-3-(2-substituirane)sukcinate in naprej $\mathrm{z}$ amoniakom ali hidroksilaminom $\mathrm{v} 2$-substituirane piridin-4,5-dikarboksilate in njihove $N$-okside. Iz teh nastanejo pri cilizaciji s hidrazinovim hidratom 7-substituirani 2,3-dihidropirido[3,4-d]piridazin-1,4-dioni in 1,4-diokso-7-substituirani 1,2,3,4-tetrahidropirido[4,3- $d]$ piridazin 6-oksidi. 